\title{
A Visual Servoing Path-Planning Strategy for Cameras Obeying the Unified Model
}

\author{
T. T. Shen, G. Chesi, and Y. S. Hung
}

\begin{abstract}
Recently, a unified camera model has been introduced in visual control systems in order to describe through a unique mathematical model conventional perspective cameras, fisheye cameras, and catadioptric systems. In this paper, a path-planning strategy for visual servoing is proposed for any camera obeying this unified model. The proposed strategy is based on the projection onto a virtual plane of the available image projections. This has two benefits. First, it allows one to perform camera pose estimation and $3 \mathrm{D}$ object reconstruction by using methods for conventional camera that are not valid for other cameras. Second, it allows one to perform image pathplanning for multi-constraint satisfaction by using a simplified but equivalent projection model, that in this paper is addressed by introducing polynomial parametrizations of the rotation and translation. The planned image trajectory is hence tracked by using an IBVS controller. The proposed strategy is validated through simulations with image noise and calibration errors typical of real experiments. It is worth remarking that visual servoing path-planning for non conventional perspective cameras has not been proposed yet in the literature.
\end{abstract}

\section{INTRODUCTION}

Visual servoing is a key area of control systems as it allows one to automatically position a robot end-point to a desired location by exploiting the information provided by a vision system (typically a camera mounted on the robot end-point) as feedback signal. Various methods have been proposed in the literature for visual servoing. Classical schemes include position-based visual servoing (PBVS) [1] and image-based visual servoing (IBVS) [2]. Other important contributions include hybrid visual servoing [3], partitioned visual servoing [4], global motion plan via navigation functions [5], and LMI techniques for multi-constraint satisfaction [6], [7]. See also the survey papers [8], [9] and the collection [10].

A fundamental problem in visual servoing consists of keeping the image features in the field of view of the camera. Various techniques have been proposed in order to address this problem, based for example on switching control schemes [11], [12], [13], circular-like trajectories [14], and path-planning in the image domain [15], [16], [17]. However, some of these techniques do not guarantee global convergence, while others do not allow one to take into account important constraints on joint limits and robot

T. T. Shen is with the Department of Electrical and Electronic Engineering, the University of Hong Kong, Pokfulam Road, Hong Kong (Email : tiantianshen@gmail.com)

G. Chesi is with the Department of Electrical and Electronic Engineering, the University of Hong Kong, Pokfulam Road, Hong Kong (Email: chesideee.hku.hk)

Y. S. Hung is with the Department of Electrical and Electronic Engineering, the University of Hong Kong, Pokfulam Road, Hong Kong (Email : yshung@eee.hku.hk) workspace. A possible way to address this problem could be to adopt vision systems with larger field of view than conventional perspective cameras, such as fisheye cameras (which combine conventional perspective cameras and fisheye lens) [18] and catadioptric imaging systems (which consist of conventional cameras and mirrors) [19]. For these vision systems, a unified mathematical model has recently been proposed and utilized in IBVS [20], [21], [22].

In this paper, an image path-planning strategy for visual servoing is proposed for cameras obeying the unified model. The idea is to reproject the available image projections onto a virtual plane. This has two benefits. First, it allows one to perform camera pose estimation and 3D object reconstruction by using methods for conventional camera that are not valid for other cameras. Second, it allows one to perform image path-planning for multi-constraint satisfaction by using a simplified but equivalent projection model, that in this paper is addressed by introducing polynomial parametrizations of the rotation and translation. The planned image trajectory is hence tracked by using an IBVS controller. Finally, the proposed strategy is validated with simulations in both cases of absence and presence of uncertainties on the image points and intrinsic parameters which are typical of real experiments. It is worth remarking that visual servoing pathplanning for non conventional perspective cameras has not been proposed yet in the literature.

The paper is organized as follows. Section II introduces the unified camera model and the problem formulation. Section III presents the proposed strategies for camera pose estimation and path-planning. Section IV shows some simulation results. Lastly, Section V concludes the paper with some final remarks.

\section{PRELIMINARIES}

In this section, we first state the notation and then we briefly recall the unified model.

\section{A. Notation}

We denote by $\mathscr{R}$ the real number set, $\mathbf{I}_{n}$ the $n \times n$ identity matrix, $\mathbf{e}_{i}$ the $i$-th column of $\mathbf{I}_{3}, \mathbf{0}_{n}$ the $n \times 1$ null vector, $\mathbf{1}_{n}$ the $n \times 1$ vector with all elements equal to $1,[\mathbf{v}]_{\times}$ the skew-symmetric matrix of $\mathbf{v} \in \mathscr{R}^{3}$. Given two camera poses $\left\{\mathbf{R}^{\circ}, \mathbf{d}^{\circ}\right\}$ and $\left\{\mathbf{R}^{*}, \mathbf{d}^{*}\right\}$, the pose transformation from $\left\{\mathbf{R}^{\circ}, \mathbf{d}^{\circ}\right\}$ to $\left\{\mathbf{R}^{*}, \mathbf{d}^{*}\right\}$ is expressed as $\{\mathbf{R}, \mathbf{d}\}$ :

$$
\left\{\begin{array}{l}
\mathbf{R}=\mathbf{R}^{\circ T} \mathbf{R}^{*} \\
\mathbf{d}=\mathbf{R}^{\circ T}\left(\mathbf{d}^{*}-\mathbf{d}^{\circ}\right) .
\end{array}\right.
$$


Pixel coordinates of images obtained from these two cameras are symbolized as $\mathbf{p}^{\circ}=\left[x^{\circ}, y^{\circ}, 1\right]^{T}$ and $\mathbf{p}^{*}=\left[x^{*}, y^{*}, 1\right]^{T}$. $K \in \mathscr{R}^{3 \times 3}$ is the camera intrinsic parameters matrix.

\section{B. Unified Camera model}

The model consists of two projections, one onto a virtual unitary sphere centered at $M$, followed by another projection from the optical center $C$ as illustrated in Fig. 1. The sphere center $M$ has a translation of $\xi$ from the optical center $C$ along the Z-axis. The unified camera model can be employed to model perspective cameras, fisheye cameras, and catadioptric systems [18].

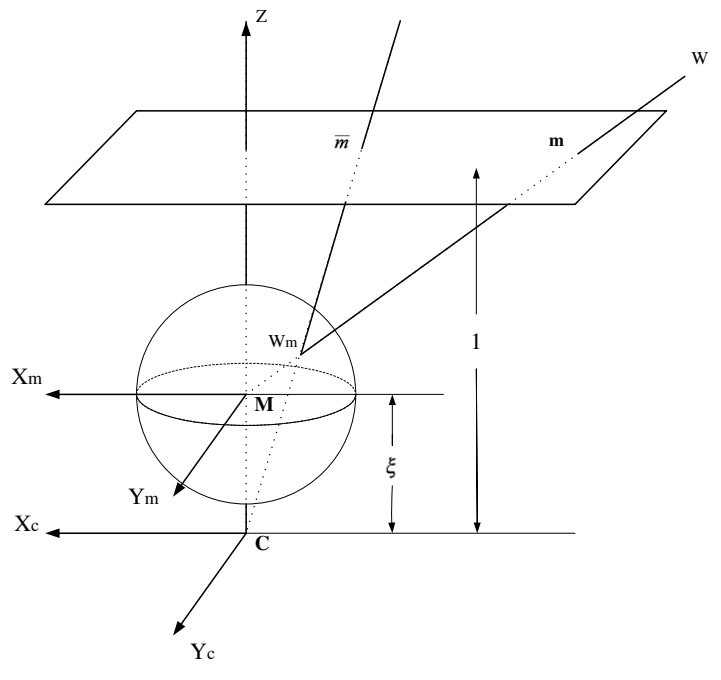

Fig. 1. Unified camera model.

Let $W$ be a 3D point with relative coordinates $W=[X, Y, Z]$ with respect to the sphere center $M$. Point $W$ is projected on to the image plane at a point with homogeneous coordinates $\overline{\mathbf{p}}=K \overline{\mathbf{m}}$, where $\overline{\mathbf{m}}$ with respect to the optical center $C$ is geometrically calculated as (See [23] for reference):

$$
\overline{\mathbf{m}}=\left[\begin{array}{ccc}
\frac{X}{Z+\xi\|W\|} & \frac{Y}{Z+\xi\|W\|} & 1
\end{array}\right]^{T} .
$$

When $\xi=0$ in (2), the unified model boils down to a conventional perspective camera. We write the projection of perspective camera as $\mathbf{p}=K \mathbf{m}$, where

$$
\mathbf{m}=\left[\begin{array}{lll}
\frac{X}{Z} & \frac{Y}{Z} & 1
\end{array}\right]^{T} .
$$

Problem. The problem we consider consists of steering the camera obeying the unified model from the initial to the desired posture satisfying visibility and workspace constraints.

\section{PROPOSED STRATEGY}

Our strategy consists of two main steps. First, we introduce a transformation for obtaining the point $\mathbf{p}$ from the point $\overline{\mathbf{p}}$, i.e. re-projecting $\overline{\mathbf{p}}$ onto the virtual plane. Second, we perform image path-planning onto the virtual plane by introducing polynomial parametrizations of the rotation and translation.

\section{A. Projection onto a Virtual Plane}

The points $\overline{\mathbf{p}}$ and $\mathbf{p}$ are related by the relationship:

$$
\overline{\mathbf{p}} \rightarrow \overline{\mathbf{m}} \rightarrow \mathbf{m} \rightarrow \mathbf{p}
$$

This relationship can be constructed by transforming $\overline{\mathbf{m}}$ into $\mathbf{m}$, which is actually a projection of $\overline{\mathbf{m}}$ onto a virtual plane according to Fig. 1. Let us write

$$
\mathbf{m}=\left[\begin{array}{lll}
m_{1} & m_{2} & 1
\end{array}\right]^{T}, \quad \overline{\mathbf{m}}=\left[\begin{array}{lll}
\bar{m}_{1} & \bar{m}_{2} & 1
\end{array}\right]^{T} .
$$

From (2) and (3), we know that $\bar{m}_{1} / \bar{m}_{2}=m_{1} / m_{2}=X / Y$. When $\bar{m}_{1} \neq 0$, for any $\bar{m}_{2}$, we let $\alpha=m_{1}=X / Z$, then $m_{2}=$ $Y / Z=(X / Z) \cdot(Y / Z)=\alpha \cdot\left(\bar{m}_{2} / \bar{m}_{1}\right)$; when $\bar{m}_{1}=0$ and $\bar{m}_{2} \neq$ 0 , we shall let $\alpha=m_{2}=Y / Z$, then $m_{1}=\alpha \cdot\left(\bar{m}_{1} / \bar{m}_{2}\right)=0$; when both $\bar{m}_{1}$ and $\bar{m}_{2}$ are zero, which happens when the object point lies in the principle axis, we have $\mathbf{m}=\overline{\mathbf{m}}=$ $[0,0,1]^{\prime}$.

$$
\begin{cases}\mathbf{m}=\left[\alpha, \alpha \bar{m}_{2} / \bar{m}_{1}, 1\right]^{T}, & \bar{m}_{1} \neq 0, \forall \bar{m}_{2} \\ \mathbf{m}=[0, \alpha, 1]^{T}, & \bar{m}_{1}=0, \bar{m}_{2} \neq 0 \\ \mathbf{m}=\overline{\mathbf{m}}, & \bar{m}_{1}=\bar{m}_{2}=0 .\end{cases}
$$

In each case mentioned above, $\mathbf{m}$ could be uniquely decided by $\overline{\mathbf{m}}$. Take the case when $\bar{m}_{1} \neq 0$ for example, by substituting $\alpha$ for $X / Z, \alpha \cdot\left(\bar{m}_{2} / \bar{m}_{1}\right)$ for $Y / Z$ in (2), we obtain:

$$
\left\{\begin{array}{l}
\bar{m}_{1}=\frac{\alpha}{1+\xi \sqrt{\alpha^{2}+\alpha^{2} \frac{\bar{m}_{2}{ }^{2}}{\bar{m}_{1}{ }^{2}}+1}} \\
\bar{m}_{2}=\frac{\alpha \cdot \bar{m}_{2} / \bar{m}_{1}}{1+\xi \sqrt{\alpha^{2}+\alpha^{2} \frac{\bar{m}_{2}{ }^{2}}{\bar{m}_{1}{ }^{2}}+1}} .
\end{array}\right.
$$

Equation (7) can be transformed into a second order polynomial of $\alpha$ :

$$
\left\{\begin{aligned}
&\left(\alpha-\bar{m}_{1}\right)^{2}=\xi^{2} \bar{m}_{1}^{2}\left(\alpha^{2}+\alpha^{2} \frac{\bar{m}_{2}^{2}}{\bar{m}_{1}^{2}}+1\right) \\
&\left(\alpha-\bar{m}_{1}\right) \bar{m}_{1}>0 .
\end{aligned}\right.
$$

It can be shown that the solution $\alpha$ of (8) is unique. From this solution, we hence recover $\mathbf{m}$ according to (6). Finally, $\mathbf{p}$ is obtained as $\mathbf{p}=K \mathbf{m}$.

\section{B. Pose Estimation and Trajectory Parametrization}

Through projection onto a virtual plane, virtual image points pairs $\left\{\mathbf{p}_{i}^{\circ}, \mathbf{p}_{i}^{*}\right\}, i=1,2, \ldots, n$ could be utilized to reconstruct the object points world coordinates $\mathbf{b}_{i}$ and to obtain the relative camera pose $\{\mathbf{R}, \mathbf{d}\}$ by solving (9) in ideal conditions:

$$
\mathbf{p}_{i}^{\circ}=\frac{K \mathbf{b}_{i}}{\mathbf{e}_{3}^{T} \mathbf{b}_{i}}, \mathbf{p}_{i}^{*}=\frac{K \mathbf{R}^{T}\left(\mathbf{b}_{i}-\mathbf{d}\right)}{\mathbf{e}_{3}^{T} \mathbf{R}^{T}\left(\mathbf{b}_{i}-\mathbf{d}\right)} .
$$

In real conditions (i.e., with image noise and calibration errors), one can estimate $\{\mathbf{R}, \mathbf{d}\}$ through essential matrix 
algorithm [24], [25] or homography matrix algorithm [26]. With approximated $\{\mathbf{R}, \mathbf{d}\}$, object coordinates $\mathbf{b}_{i}$ can be estimated using linear least square method. When the CAD model of the object is not available, the estimated $\mathbf{d}$ and the planned camera trajectory will be normalized [16].

Let us denote with $\{\mathbf{R}(w), \mathbf{d}(w)\}$ the camera pose along the trajectory, where $w \in[0,1]$ is the trajectory abscise. The pose boundary conditions are:

$$
\left\{\begin{array}{l}
\{\mathbf{R}(0), \mathbf{d}(0)\}=\left\{\mathbf{I}_{3}, \mathbf{0}_{3}\right\} \\
\{\mathbf{R}(1), \mathbf{d}(1)\}=\{\mathbf{R}, \mathbf{d}\} .
\end{array}\right.
$$

The image boundary conditions are given by:

$$
\left\{\begin{array}{l}
\overline{\mathbf{p}}_{i}(w)=\frac{K \mathbf{R}(w)^{T}\left(\mathbf{b}_{i}-\mathbf{d}(w)\right)}{\mathbf{e}_{3}^{T} \mathbf{R}(w)^{T}\left(\mathbf{b}_{i}-\mathbf{d}(w)\right)+\xi\left\|\left(\mathbf{b}_{i}-\mathbf{d}(w)\right)\right\|} \\
\overline{\mathbf{p}}_{i}(0)=\overline{\mathbf{p}}_{i}^{\circ}, \overline{\mathbf{p}}_{i}(1)=\overline{\mathbf{p}}_{i}^{*}, \forall i=1, \ldots, n
\end{array}\right.
$$

where $\mathbf{b}_{i}$ is reconstructed world coordinates of the $i$-th object point. However, in real conditions, there does not exist any $\mathbf{b}_{i}$ such that $\overline{\mathbf{p}}_{i}(0)=\overline{\mathbf{p}}_{i}^{\circ}$ and $\overline{\mathbf{p}}_{i}(1)=\overline{\mathbf{p}}_{i}^{*}$ due to image noise and calibration errors, and therefore we impose that:

$$
\overline{\mathbf{p}}_{i}(w)=\overline{\mathbf{p}}_{i}(w)-(1-w) \varepsilon_{i}^{\circ}-w\left(\varepsilon_{i}^{*}\right) .
$$

where $\varepsilon_{i}^{\circ}$ and $\varepsilon_{i}^{*}$ are image errors that ensure the feasibility of the problem, and are given by:

$$
\left\{\begin{array}{l}
\varepsilon_{i}^{\circ}=\frac{K \mathbf{b}_{i}}{\mathbf{e}_{3}^{T} \mathbf{b}_{i}+\xi\left\|\mathbf{b}_{i}\right\|}-\overline{\mathbf{p}}_{i}^{\circ} \\
\varepsilon_{i}^{*}=\frac{K \mathbf{R}^{T}\left(\mathbf{b}_{i}-\mathbf{d}\right)}{\mathbf{e}_{3}^{T} \mathbf{R}^{T}\left(\mathbf{b}_{i}-\mathbf{d}\right)+\xi\left\|\left(\mathbf{b}_{i}-\mathbf{d}\right)\right\|}-\overline{\mathbf{p}}_{i}^{*} .
\end{array}\right.
$$

In order to deal with polynomial optimization, we represent the rotation matrix $\mathbf{R}(w)$ in the form of $\Lambda(\phi(w))$, where $\phi(w) \in \mathscr{R}^{4}$ are quaternions [16]. The desired quaternion $\phi(1)$ corresponding to the desired rotation $\mathbf{R}$ is denoted as $\phi^{*}$ :

$$
\phi^{*}=\left[\sin \frac{\theta}{2} \mathbf{v}^{T}, \cos \frac{\theta}{2}\right]^{T}
$$

where $\theta \in[0, \pi]$ and $\mathbf{v} \in \mathscr{R}^{3}:\|\mathbf{v}\|=1$, are respectively the rotation angle and axis of $\mathbf{R}$, which can easily be found when $\mathbf{R}$ is expressed in its exponential coordinates form as $\mathbf{R}=e^{[\theta \mathbf{v}] \times}$. Hence, the camera pose along the trajectory is represented by $\{\phi(w), \mathbf{d}(w)\}$ and the pose boundary conditions in (10) is newly described as:

$$
\left\{\begin{array}{l}
\{\phi(0), \mathbf{d}(0)\}=\left\{\left[\mathbf{0}_{3}, 1\right]^{T}, \mathbf{0}_{3}\right\} \\
\{\phi(1), \mathbf{d}(1)\}=\left\{\boldsymbol{\phi}^{*}, \mathbf{d}\right\} .
\end{array}\right.
$$

Polynomial parametrization of $\phi(w)$ and $\mathbf{d}(w)$ are employed to reduce the computational complexity. We write them in polynomials of $w$ with arbitrary degrees of $\sigma$ and $\tau$ respectively:

$$
\left\{\begin{array}{l}
\phi(w)=\tilde{U} \cdot\left[w^{\sigma}, w^{\sigma-1}, \ldots, w, 1\right]^{T} \\
\mathbf{d}(w)=\tilde{V} \cdot\left[w^{\tau}, w^{\tau-1}, \ldots, w, 1\right]^{T}
\end{array}\right.
$$

where $\phi(w) \in \mathscr{R}^{4}$ and $\mathbf{d}(w) \in \mathscr{R}^{3}$, therefore their coefficient matrices $\tilde{U} \in \mathscr{R}^{4 \times(\sigma+1)}$ and $\tilde{V} \in \mathscr{R}^{3 \times(\tau+1)}$. It is a difficult problem to determine the optimal polynomial degree. We shall select these polynomial degrees from low to high until a satisfactory trajectory is obtained. By imposing boundary conditions on $\phi(w)$ and $\mathbf{d}(w)$ in (16), one obtains

$$
\left\{\begin{array}{l}
\tilde{U}=\left[\boldsymbol{\phi}^{*}-U \cdot \mathbf{1}_{\sigma-1}-\left[\mathbf{0}_{3}^{T}, 1\right]^{T}, U,\left[\mathbf{0}_{3}^{T}, 1\right]^{T}\right], \\
\tilde{V}=\left[\mathbf{d}-V \cdot \mathbf{1}_{\tau-1}, V, \mathbf{0}_{3}\right]
\end{array}\right.
$$

where $U \in \mathscr{R}^{4 \times(\sigma-1)}$ and $V \in \mathscr{R}^{3 \times(\tau-1)}$ should be optimized to satisfy the imposed constraints.

\section{Constraints}

It is compulsory for the robot to keep targets in its field of view during the motion. Occlusions should be avoided as done is [17]. At the same time, the robot should also avoid collision with obstacles lie in the workspace. In this section, we impose visibility constraint and workspace constraint on the camera trajectory.

1) Visibility Constraint: In order to keep sight of objects along the trajectory of the camera, the objects should always be in front of the camera and the image projection of the objects should be constrained within the fixed image size $\bar{\zeta}_{x} \times \bar{\zeta}_{y}$. These constraints are expressed as:

$$
\left\{\begin{array}{l}
0 \leq x_{i}(w) \leq \zeta_{x} \\
0 \leq y_{i}(w) \leq \zeta_{y} \\
Z_{i}(w)>0, \forall w \in[0,1], i=1, \ldots, n
\end{array}\right.
$$

where $\zeta_{x}$ and $\zeta_{y}$ are virtual image size on the virtual plane corresponding to the real image size of $\bar{\zeta}_{x} \times \bar{\zeta}_{y}, x_{i}(w)$ and $y_{i}(w)$ are image points projection onto the virtual plane, $Z_{i}(w)$ is parameterized object point depth:

$$
\begin{aligned}
& \mathbf{p}=\left[\begin{array}{lll}
x_{i}(w) & y_{i}(w) & 1
\end{array}\right]^{T} \\
& =\frac{K \Lambda(\phi(w))^{T}\left(\mathbf{b}_{i}-\mathbf{d}(w)\right)}{Z_{i}(w)} \\
& Z_{i}(w)=\mathbf{e}_{3}^{T} \Lambda(\phi(w))^{T}\left(\mathbf{b}_{i}-\mathbf{d}(w)\right) .
\end{aligned}
$$

For catadioptric sensor, the dead zone at the center of the image should also be considered, we denote $\zeta_{o}$ the projection of the dimension of the dead zone onto a virtual plane and $\left\{x_{o}, y_{o}\right\}$ the image center.

$$
\left[x_{i}(w)-x_{o}\right]^{2}+\left[y_{i}(w)-y_{o}\right]^{2} \geq \zeta_{o} .
$$

In catadioptric systems, both (18) and (21) should be considered. It can be noticed that all these constraints are polynomial inequalities. For perspective cameras, only (18) should be satisfied. When fisheye camera with $\xi=1$ in the unified model is used, the point depth constraint is enough to ensure the visibility. In the sequel, we show in detail the point depth constraint for a fisheye camera. Bring polynomial parametrization of $\phi(w)$ and $\mathbf{d}(w)$ in (20), we obtain: 


$$
Z_{i}(w)=\left[w^{\tau}, w^{\tau-1}, \ldots, w, 1\right] \cdot M_{\text {coef }} \cdot\left[w^{2 \sigma}, w^{2 \sigma-1}, \ldots, w, 1\right]^{T}
$$

where

$$
\begin{aligned}
& M_{\text {coef }}=2 \mathbf{r}_{1}\left[\tilde{\mathbf{u}}_{3}^{T} \tilde{\mathbf{u}}_{1}+\tilde{\mathbf{u}}_{4}^{T} \tilde{\mathbf{u}}_{2}\right]_{\oslash}+2 \mathbf{r}_{2}\left[\tilde{\mathbf{u}}_{3}^{T} \tilde{\mathbf{u}}_{2}-\tilde{\mathbf{u}}_{4}^{T} \tilde{\mathbf{u}}_{1}\right]_{\oslash} \\
& +\mathbf{r}_{3}\left[\tilde{\mathbf{u}}_{1}^{T} \tilde{\mathbf{u}}_{1}-\tilde{\mathbf{u}}_{2}^{T} \tilde{\mathbf{u}}_{2}+\tilde{\mathbf{u}}_{3}^{T} \tilde{\mathbf{u}}_{3}+\tilde{\mathbf{u}}_{4}^{T} \tilde{\mathbf{u}}_{4}\right]_{\oslash} .
\end{aligned}
$$

In (23), $\mathbf{r}_{i} \in \mathscr{R}^{(\tau+1) \times 1}$ is the $i$-th column of matrix $\left[V \cdot \mathbf{1}_{\tau-1}-\right.$ $\left.\mathbf{d},-V, \mathbf{b}_{i}\right]^{T}, \tilde{\mathbf{u}}_{i} \in \mathscr{R}^{1 \times(\sigma+1)}$ is the $i$-th row of matrix $\tilde{U}$ in (17), and $[H]_{\oslash}$ is a $1 \times(2 \sigma+1)$ vector whose $\rho$-th component is the sum of entries $H_{i, j}$ that satisfies $i+j=\rho+1,1 \leq i, j \leq$ $\sigma+1$ in matrix $H \in \mathscr{R}^{(\sigma+1) \times(\sigma+1)}$. From (22) and (23), we can see that $Z_{i}(w)$ is a polynomial of $w$ with degree of $2 \sigma+$ $\tau$. The optimization problem lies in finding the appropriate matrices $U$ and $V$ that satisfy the point depth constraint for every $i=1, \ldots, n$ and $w \in[0,1]$ along the trajectory.

2) Workspace Constraint: Robot should make a detour to avoid collision with obstacles block in the way, at the same time it should keep sight of all the object points.

Assume that there is an obstacle located at $\mathbf{o} \in \mathscr{R}^{3}$ with reference to the initial camera pose, one can impose workspace constraint on the camera trajectory as:

$$
\|\mathbf{d}(w)-\mathbf{o}\| \geq a, \forall w \in[0,1]
$$

where $a$ is a constant describing the minimum safe distance between camera trajectory $\mathbf{d}(w)$ and the obstacle. Bring in polynomial parametrization of $\mathbf{d}(w)$, we have

$$
\left\|\left[\mathbf{d}-V \cdot \mathbf{1}_{\tau-1}, V,-\mathbf{o}\right] \cdot\left[w^{\tau}, w^{\tau-1}, \ldots, w, 1\right]^{T}\right\|>a .
$$

\section{Getting the Trajectory}

All constraints should be satisfied by solving the optimization problem:

$$
\begin{aligned}
& g_{k}(w, U, V, i)>0, \\
& \forall k=1, \ldots, k_{M A X}, \forall w \in[0,1], \forall i=1, \ldots, n .
\end{aligned}
$$

It must be evaluated that each constraint function $g_{k}(w), k=1, \ldots, k_{M A X}$ is positive for all $w \in[0,1]$ and all $i=1, \ldots, n$ which is the sequence of observable object points.

In the sequel, we focus on the utilization of fisheye cameras, in which case only the point depth constraint should be considered to ensure the visibility. Entries of $U$ and $V$ matrices are initially set to be zero, that generate a straight trajectory. With initial $U$ and $V$, we first find $g_{1 i}$, the minimum value of $Z_{i}(w)$ along the trajectory for the $i$ th object point, then obtain $g_{1}$, the minimum value of $g_{1 i}$ among different object points.

$$
\begin{aligned}
g_{1 i} & =\min _{w \in[0,1]} Z_{i}(w) \\
g_{1} & =\min _{i=1, \ldots, n} g_{1 i} .
\end{aligned}
$$

If $g_{1}$ is positive, then the visibility constraint is already satisfied, otherwise we should start the optimization. In order to impose workspace constraint simultaneously, let

$$
g_{2}=\min _{w \in[0,1]}\|\mathbf{d}(w)-\mathbf{o}\|-a .
$$

Put these two constraints together, we define:

$$
G(Q)=\min \left\{g_{1}, g_{2}\right\}
$$

where $Q$ is a vector containing all the entries of $U$ and $V$. The problem is:

$$
G^{*}=\max _{Q} G(Q) .
$$

The solution of (30) may not be unique since the imposed constraints may be satisfied by different trajectories. One can simply adopt the solution returned by the solver. Since $U$ and $V$ are united as a vector $Q$, local maximum of $G(Q)$ is guaranteed. We denote the appropriate $U$ and $V$ as $\hat{U}$ and $\hat{V}$.

Then the camera trajectory can be obtained by imposing $\hat{U}$ and $\hat{V}$ in (16) and plotting $\mathbf{d}(w)$ with high resolution of w. Image trajectory can be obtained by:

$$
\overline{\mathbf{p}}_{i}(w)=\frac{K \Lambda(\phi(w))^{T}\left(\mathbf{b}_{i}-\mathbf{d}(w)\right)}{\mathbf{e}_{3}^{T} \Lambda(\phi(w))^{T}\left(\mathbf{b}_{i}-\mathbf{d}(w)\right)+\xi\left\|\mathbf{b}_{i}-\mathbf{d}(w)\right\|} .
$$

Image trajectory on the virtual plane is nothing but the image trajectory obtained by (31) when $\xi=0$.

\section{EXAMPLES}

In this section, the proposed path-planning strategy for cameras obeying the unified model is demonstrated by simulation results. The performance of fisheye camera (with $\xi=1$ in the unified model) and perspective one are compared in these simulation examples. It takes about 5.61 seconds to find $\hat{U}$ and $\hat{V}$ on a standard PC (Intel Core 2 Duo, WIN $\mathrm{XP}, 2.10 \mathrm{GHz}$ ) with MATLAB. It is supposed that the CAD model of the object is not available and the camera has its ideal intrinsic parameters matrix as:

$$
K=\left(\begin{array}{ccc}
400 & 0 & 400 \\
0 & 400 & 300 \\
0 & 0 & 1
\end{array}\right)
$$

Consider the situation depicted in Fig. 2 where the large white dots of five dices are observed by two fisheye cameras, the initial one at $[0,0,0]^{T}$ with rotation axis $\mathbf{v}=[0,0,1]^{T}$ and rotation angle $\theta=0$, and the desired one at $[-22,0,60]^{T}$ with rotation axis $\mathbf{v}=[0,1,0]^{T}$ and rotation angle $\theta=2.1991$.

Consider the problem to steer the camera satisfying visibility and workspace constraints simultaneously. When there is an obstacle demonstrated as a sphere right on the straight line between these two camera centers, the workspace constraint will not be satisfied if $U$ and $V$ maintain their initial values. The camera has to make a detour to avoid collision. Fig. 3 and 4 show the path-planning results through optimization.

In real conditions, we assume random image noise range from $[-1,1]$ and the estimated intrinsic parameters matrix $\tilde{K}$ as: 


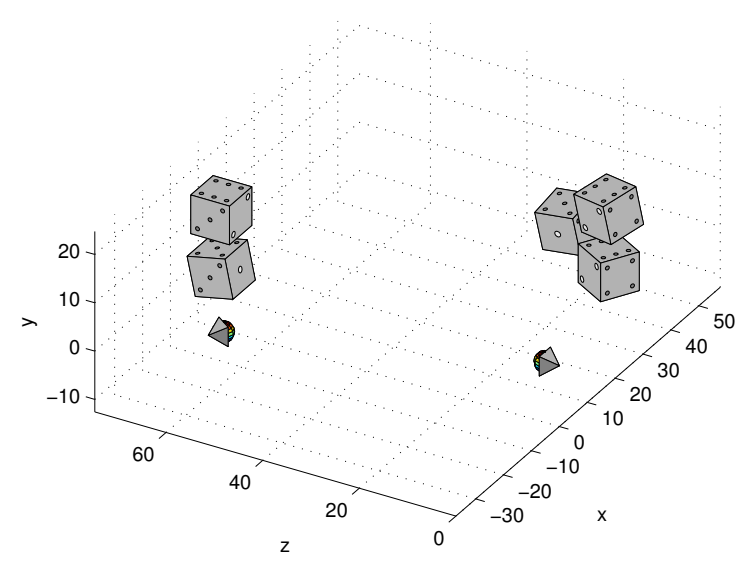

Fig. 2. 3D points and cameras.

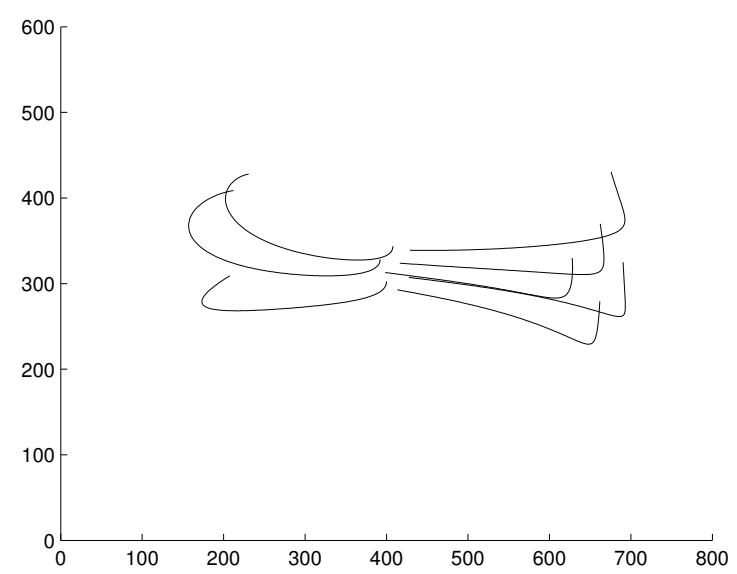

Fig. 3. Image trajectory of fisheye projection in ideal condition.

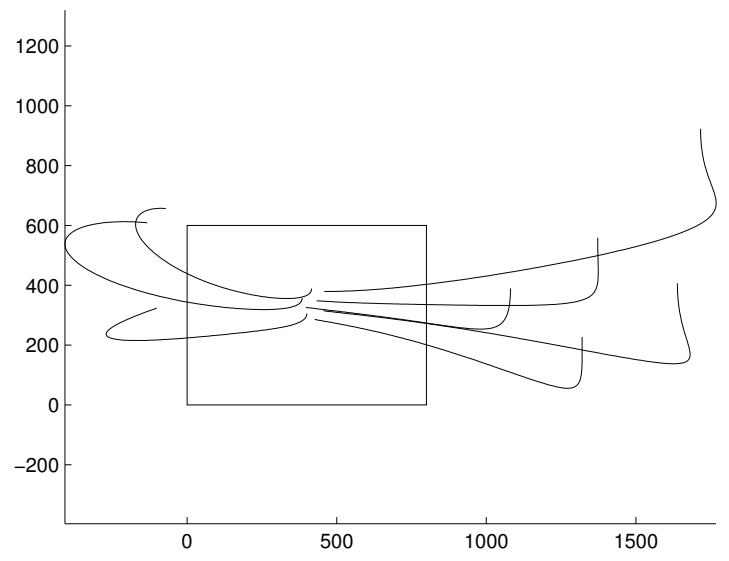

Fig. 4. Image trajectory onto the virtual plane in ideal condition.

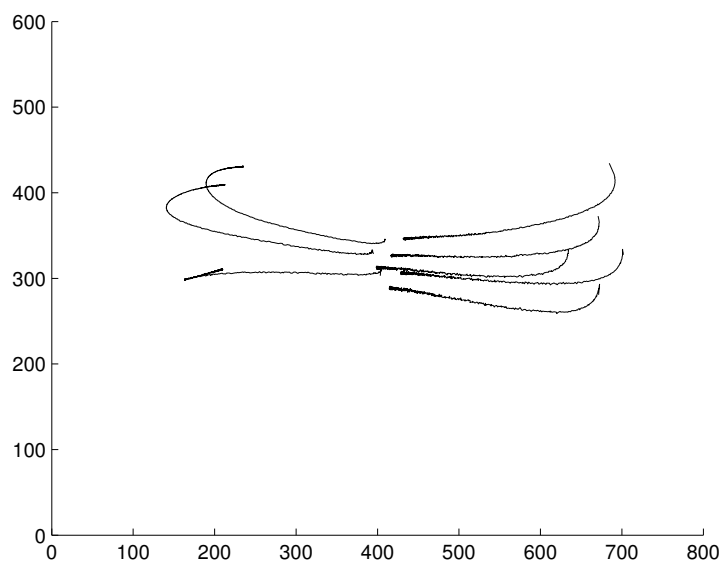

Fig. 5. Image trajectory of fisheye projection in real condition.

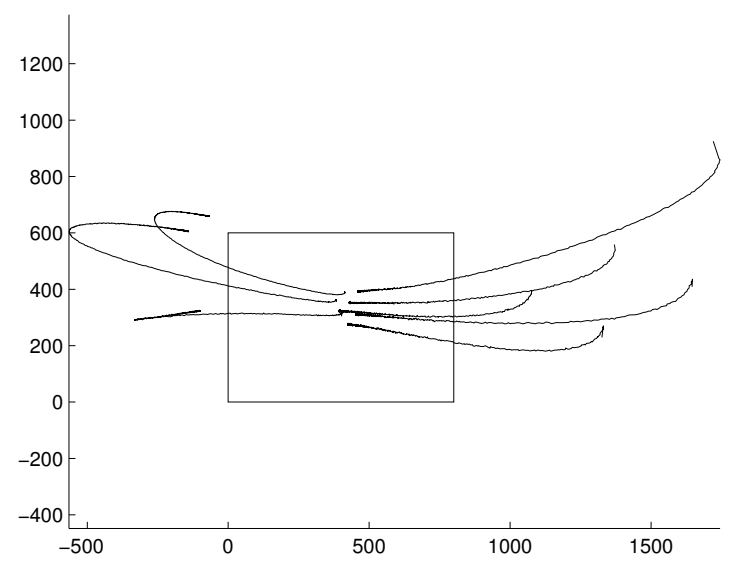

Fig. 6. Image trajectory onto the virtual plane in real condition.

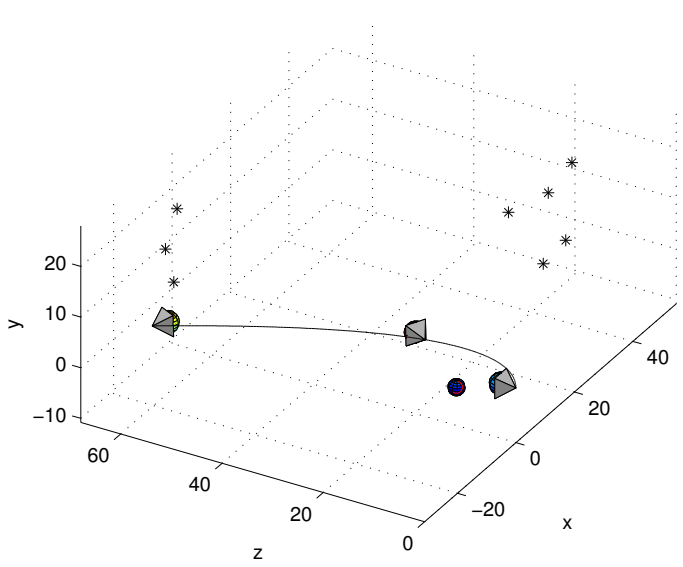

Fig. 7. Camera path in real condition. 


$$
\tilde{K}=\left(\begin{array}{ccc}
412 & 0 & 405 \\
0 & 390 & 302 \\
0 & 0 & 1
\end{array}\right) .
$$

The path-planning results with image noise and calibration errors are shown in Fig. 5, 6 and 7.

Fig. 3 and 5 indicate that image trajectory of fisheye camera falls within the fixed image size of $800 \times 600$ pixels. For comparison, image trajectory of projection onto the virtual plane shown in Fig. 4 and Fig. 6 apparently goes out of the image boundary presented as a rectangle.

Fig. 7 shows the planned camera path in real conditions. Camera pose in the middle of the planned path with trajectory parameter $w=0.5$ is also figured in Fig. 7 .

\section{CONCLUSIONS AND FUTURE WORKS}

We have presented a visual path-planning strategy for cameras obeying the unified model, which includes conventional perspective cameras, fisheye cameras, and catadioptric systems. The idea consists of re-projecting the available image projections onto a virtual plane, hence allowing one to perform image path-planning for multi-constraint satisfaction by using a simplified but equivalent projection model. In addition, the proposed strategy also allows one to perform camera pose estimation and 3D object reconstruction by using methods for conventional camera that are not valid for other cameras. It is worth remarking that visual servoing path-planning for non conventional perspective cameras has not been proposed yet in the literature. Future work will be devoted to test the behavior of the proposed strategy in real experiments.

\section{ACKNOWLEDGMENTS}

The authors would like to thank the Associate Editor and the Reviewers for their useful comments. This work was supported in part by the Research Grants Council of Hong Kong (Grants HKU711208E and HKU712808E).

\section{REFERENCES}

[1] C. Taylor and J. Ostrowski, "Robust vision-based pose control", Proc. IEEE Int. Conf. Robot. Automat., San Francisco, CA, pp. 2734-2740, 2000.

[2] K. Hashimoto, T. Kimoto, T. Ebine, and H. Kimura, "Manipulator control with image-based visual servo", Proc. IEEE Int. Conf. Robot. Automat., pp. 2267-2272, 1991.

[3] E. Malis, F. Chaumette, and S. Boudet, "2 1/2 D visual servoing", IEEE Trans. Robot. Automat., vol. 15, no. 2, pp. 238-250, Apr. 1999.

[4] P. Oh and P. Allen, "Visual servoing by partitioning degrees of freedom", IEEE Trans. Robot. Automat., vol. 17, no. 1, pp. 1-17, Feb. 2001.

[5] N. Cowan, J. Weingarten, and D. Koditschek, "Visual servoing via navigation functions", IEEE Trans. Robot. Automat., vol. 18, no. 4, pp. 521-533, Aug. 2002.

[6] P. Danes, D. F. Coutinho, and S. Durola, "Multicriteria Analysis of Visual Servos through Rational Systems, Biquadratic Lyapunov Functions, and LMIs", in G. Chesi and K. Hashimoto (Eds), Visual Servoing via Advanced Numerical Methods, Springer, 2010.

[7] S. Tarbouriech and P. Soueres, "Image-based Visual Servo Control Design with Multi-Constraint Satisfaction", in G. Chesi and K. Hashimoto (Eds), Visual Servoing via Advanced Numerical Methods, Springer, 2010.
[8] F. Chaumette and S. Hutchinson, "Visual Servo Control, Part I: Basic Approaches", IEEE Robotics and Automation Magazine., vol. 13, no. 4, pp. 82-90, 2006.

[9] F. Chaumette and S. Hutchinson, "Visual Servo Control, Part II: Advanced Approaches", IEEE Robotics and Automation Magazine., vol. 14, no. 1, pp. 109-118, 2007.

[10] G. Chesi and K. Hashimoto, editors. "Visual Servoing via Advanced Numerical Methods", Springer, 2010.

[11] G. Chesi, K. Hashimoto, D. Prattichizzo, and A. Vicino, "Keeping Features in the Field of View in Eye-In-Hand Visual Servoing: A Switching Approach", IEEE Trans. Robot., vol. 20, no. 5, Oct. 2004.

[12] N. R. Gans and S. A. Hutchinson, "Stable Visual Servoing Through Hybrid Switched-System Control," IEEE Trans. Robot., vol.23, no.3, pp. 530-540, Jun. 2007.

[13] G. Lopez-Nicolas, C. Sagues, J. J. Guerrero, D. Kragic, and P. Jensfelt, "Switching visual control based on epipoles for mobile robots," Robotics and Autonomous Systems., vol. 56, iss. 7, pp. 592-603, July 2008.

[14] G. Chesi and A. Vicino, "Visual servoing for large camera displacements," IEEE Trans. on Robotics., vol. 20, no. 4, pp. 724C735, 2004.

[15] Y. Mezouar and F. Chaumette, "Path planning for robust image-based control". IEEE Trans. on Robotics and Automation., 18(4): 534-549, 2002.

[16] G. Chesi and Y. S. Hung, "Global Path-Planning for Constrained and Optimal Visual Servoing", IEEE Trans. Robot., vol. 23, no. 5, Oct. 2007.

[17] G. Chesi, "Visual servoing path-planning via homogeneous forms and LMI optimizations", IEEE Trans. Robot., vol. 25, no. 2, pp. 281-291, 2009.

[18] J. Courbon, Y. Mezouar, L. Eck, and P. Martinet, "A Generic Fisheye Camera Model for Robotic Applications", IEEE Int. Conf. on Intelligent Robots and Systems., San Diego, CA, pp. 1683-1688, 2007.

[19] G. L. Mariottini, S. Scheggi, F. Morbidi, and D. Prattichizzo, "Catadioptric Stereo with Planar Mirrors: Multiple-view Geometry and Camera Localization", in G. Chesi and K. Hashimoto (Eds), Visual Servoing via Advanced Numerical Methods, Springer, 2010.

[20] C. Geyer and K. Daniilidis, "A unifying theory for central panoramic systems and practical implications: A Switching Approach", Eur. Conf. on Computer Vision., vol. 29, no. 3, pp. 159-179, May 2000.

[21] X. Ying and Z. Hu, "Can we consider central catadioptric cameras and fisheye cameras within a unified imaging model", Eur. Conf. on Computer Vision., vol. 1, pp. 442-455, 2004.

[22] O. Tahri, Y. Mezouar, F. Chaumette, and H. Araujo, "Visual Servoing and Pose Estimation with Cameras Obeying the Unified Model", in G. Chesi and K. Hashimoto (Eds), Visual Servoing via Advanced Numerical Methods, Springer, 2010.

[23] O. Tahri, Y. Mezouar, F. Chaumette, and P. Corke, "Generic Decoupled Image-Based Visual Servoing for Cameras Obeying the Unified Projection Model", IEEE Int. Conf. Robotics and Automation., pp. 1116-1121, 2009.

[24] J. Y. Weng, T. S. Huang, and N. Ahuja, "Motion and Structure from Two Perspective Views: Algorithms, Error Analysis, and Error Estimation”. IEEE Trans. on Pattern Analysis and Machine Intelligence., vol. 11, no. 5, May 1989.

[25] G. Chesi, "Camera Displacement via Constrained Minimization of the Algebraic Error". IEEE Trans. On Pattern Analysis And Machine Intelligence, vol. 31, no. 2, pp. 370-375, Feb. 2009.

[26] E. Malis and F. Chaumette, " 2 1/2 D visual servoing with respect to unknown objects through a mew estimation scheme of camera displacement", Int. J. Comput. Vis., vol. 37, no. 1, pp. 79-97, 2000. 